\title{
A NEW GATE DRIVE CIRCUIT FOR MOSFETS SWITCHING AT LOW FREQUENCY
}

\author{
S.R. Narayana Prakash \\ B.S.R. Iyengar \\ Department of Electrical Engg. \\ Indian Institute of Science \\ Bangalore 560012 , INDIA
}

P.V. Ananda Mohan

Digital Group, Transmission R\&D Indian Telephone Industries Ltd. Bangalore 560016 , INDTA

\section{ABSTRACT}

A new gate drive circuit, with ferrite core transformer isolation, for power MOSFETS switching a tow frequency in the micro Hertz range has been presented. The technique involves periodic refreshing of charge on the effective gate source capacitance of the MOSFET in order to ensure low $\mathrm{ON}-$ state resistance, $\mathrm{R}_{\mathrm{S}}(\mathrm{ON})$, by maintaining its gate-source voltage above the threshold level. The simulation of the drive circuit using circuit analysis program PSPICE is described with results arid relevant waveforms.

\section{INTRODUCTION}

The availability of reliable and efficient power semiconductor switching devices has contributed significantly to the development and application of power electronic systems in the fields of generation, transmission and distribution of electric power telecommunication and variable speed drives. The direction, extent and pace of progress in the development and application of power electronics in these areas has been dependent largely on the improvements in the power handling capability as well. as the control schemes of these switching devices. The selection of these devices, such as thyristors (SCRs) bipolar transistors (BJTs), MOSFETs and isolated gate bipolar transistors (IGBTs), is decided by the forward current, blocking voltage, turn-on \& turn-off times and tho switching losses that the device is expected to handle in a given application. Thyristors are used for low frequency application due to its considerable turn-on, turn-off times and involved commutation process. For high frequency switching applications BJTs, MOSFETs and IGBTs are preferred on account of their excellent switching characteristics like negligible turn-on and turn-off times anc easier control schemes. In general, most of these switching devices are provided with isolated drive using pulse transformers, for isolating the power and control circuits.

Therefore, when the switching frequency is extremely low and devices such as BJTs, MOSFETs and IGBTs are used as controlled switches with isolated drive, the size of the pulse transformer becomes enormously large for preventing core saturation. Further, the BJTs consume considerable drive power compared to MOSFETs under similar operating conditions. To alleviate these problems, a new ferrite cored transformer coupled gate drive circuit has been developed for power MOSFETs switching a t low frequency incorporating features like negligible turn-on and turn-off times, low gate power requirement, and miniature ferrite cored pulse transformer. This new gate drive circuit finds applications in switch mode DC-DC converters and DC-AC inverters with high frequency $1 \mathrm{ink}$ and PMM control [6-8] wherein some of the MOSFETs have to condust with duty cycle extended beyond $50 \%$.

\section{PRINCIPLE OF OPERATION}

The power MOSEETs are charge controlled devices [1-4] with a nearly pure capacitance as the input impedance between its gate and source. For a N-channel enhancement mode MOSPET to switch with low on-state resistance ${ }_{\mathrm{R}}(\mathrm{ON})$, its effective gate source capacitance CS (eff) must be charged with a gate drive such that the voltage $V_{\text {across }}$ at, is above the threshold level V GS (typically 10 volts). In the absence of such a gate drive, the loss of charge on $\mathrm{C}_{\text {(eff) due to leakage current leads }}$ to the vol Eage across it to fall exponentially

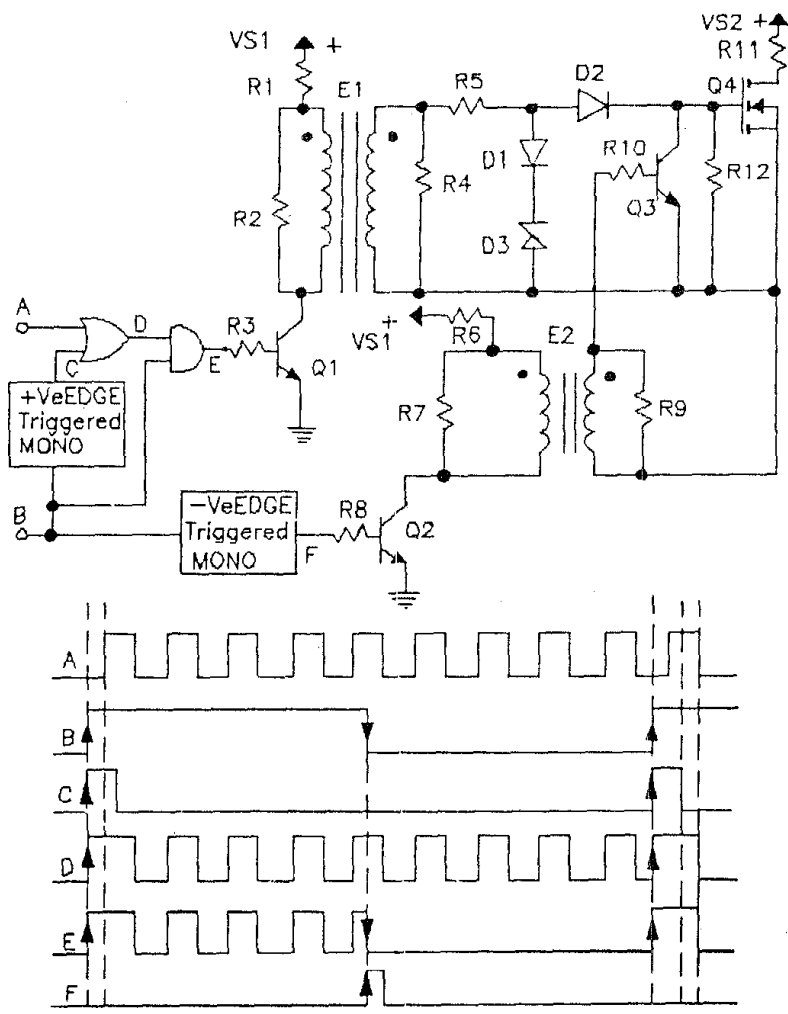

Figure 1: New Gate Drive Circuit for Enhancement Type N-Channel MOSFET with Extended Duty Cycle 
below $V_{T C}$ and consequent increase of the onstate rêtistance. $R_{\text {Ts }}(O N)$. Thus in order to maintain the RDS(ON) low, it is mandatory to refresh the charge on $\mathrm{C}_{\text {foff }}$ (ef a a rate greater than the rate at which the charge is lost due to the leakage current. When the MOSFET has to be maintained in the, ON-state with low $R_{\text {S }}(\mathrm{ON})$ for long duration, it would be impossible Lo maintain the ferrite cored pulse transformer, to remain unsaturated with a constant drive pulse such that $V_{G S}$ is above $V_{T O}$ at any specified drain current, GS drain sourde voltage and operating temperature. To circumvent this problem, the charge on the effective gate source capacitance is refreshed at high frequency (above $20 \mathrm{kHz}$ ) such that the voltage acrose $C_{G S}$ (eff) is above the gate threshold voltage $V_{\text {To }}$ for the duration of conduction and the fextite transformer remains unsaturated.

The concept of periodic refreshing of charge used in DRAMs to retain its memory [23], has teen extended in this paper for control. of POWER MOSEETs as an ideal controlled switch at low frequency. The proposed gate drive circuit schematic is shown in Figure 1. The POWER MOSFET Q4 is the switch to be operated a low frequency and the duration of conduction of which is decided by pulse B. The resistor R12 (which represents the resistance of the leakage path) and the effective gate source capacitance

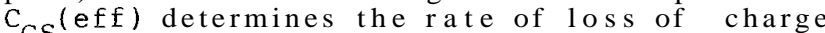
ons C C (eff). The charge on $C_{G S}$ (eff) is refresked at a rate faster than, the rate of discharge by pulse A via the transistor Ql, ferrite cored pulse tradsformer El, R5 and blocking diode D2, such that V is above VTO for the duration of conduction of MOSFET Q4, Q3 is maintained in the OFF - state. The diode D1 and the zener diode D3 limit the voltage V approximately to that of the zener diods voltage rating. The diode D2 prevents the gate-source capacitance from discharging through the transformer El in the absence of a charging pulse, while Dl prevents the zener disde D3 from conducting during the off time. For Q4 to turn-on when B goes high, the first rising edge of A must. be synchronised with the rising edge of B. In situations wherein this condition is not met by A, the output of the positive edge triggered monostable multivibrator i.e., pulse C, in combination with A provides the required pulse D which satisfies the above mentioned criterion. The width of pulse C preferably must not exceed $1 / 4$ th the period of pulse $A$ and the transformer El must be designed to operate without saturation with extended pulse widths as shown by pulse D. In order to minimise the size of the ferrite cored pulse transformer El, the frequency of $A$ is fixed above $20 \mathrm{kHz}$. The duration of refreshing of charge on $\mathrm{C}_{G S}$ (eff) via Qland El is controlled by $B$ as shown by pulse E. The MOSFET Q4 is turned off by discharging $\mathrm{C}_{\text {( }}$ (eff). This is accomplished by

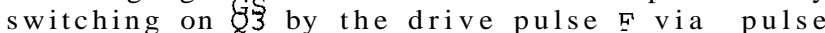
transformer E2 and Q2. The turn off pulse $F$ is generated a the end of the duration of conduction by the negative edge triggered monostable multivibrator when B goes low.

The voltage source vsl provides the required energy to charge and discharge the gate source capacitance. The voltage sourc

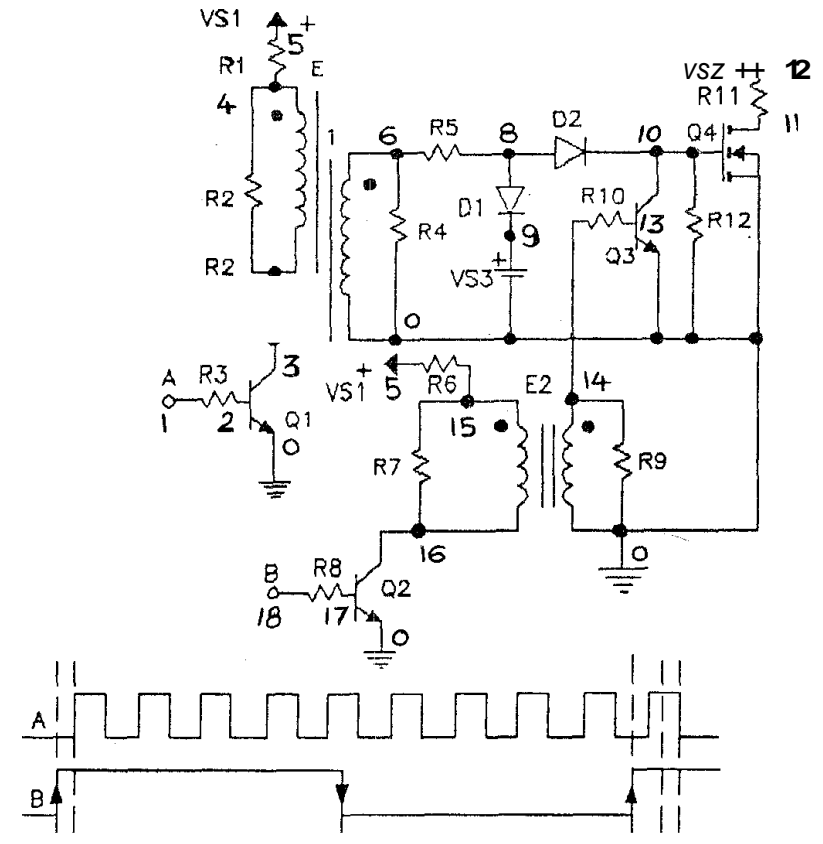

Figure 2: Equivalent Circuit of New Gate Drive for Simulation using PSPICE.

VS2 along with load resistor Rll determines the drain current. The prototype of the new gate drive circuit has been tested with BUZ 24 power MOSFET. It has been observed that a turn on time of 0.6 microsec. and a turn off time of 0.2 microsec can be achieved with negligible gate power consumption.

\section{SIMULATION WIIH PSPICE}

The new gate drive circuit has been simulated using the circuit analysis program PSPICE on a personal computer. The equivalent circuit for simulation is shown in Figure 2. For the purpose of simplification, the various logic gates shown in Figure 1 have been eliminated. The gate source capacitance is charged by pulse A via Q1, E1, R5 and D2. The frequency of $A$ is fixed a $25 \mathrm{kHz}$ with $50 \%$ duty ratio. The transformer El is the voltage controlled voltage source with 6 and 0 as controlled nodes, 4 and 3 a s controlling nodes and voltage transfer ratio being $v_{43} / v_{60}=1 / 1.2$. The zener diode D3 is rêplaced by a constant DC voltage source VS3 of 15 volts. The leakage current is determined by resistor R12 at a given V The effective gate source capacitance GGSO for Q4 is represented by a channel width of 2 microns and a gate source overlap capacitance of 750 micro Farads per meter of channel width [5]. The gate source threshold voltage, for Q4 to be in the ON-state, is set by VIO (typically 10.0 volts). The maximum forward current gain Beta is fixed at 100.0 for the BJTs. The pulse transformer E2 is represented by another voltage controlled voltage source with 14 and 0 as the controlled nodes, 15 and 16 as controlling nodes and voltage cransier ratio being $\mathrm{V}_{15,16} / \mathrm{V}_{14,0}=1 / 1.2$. 

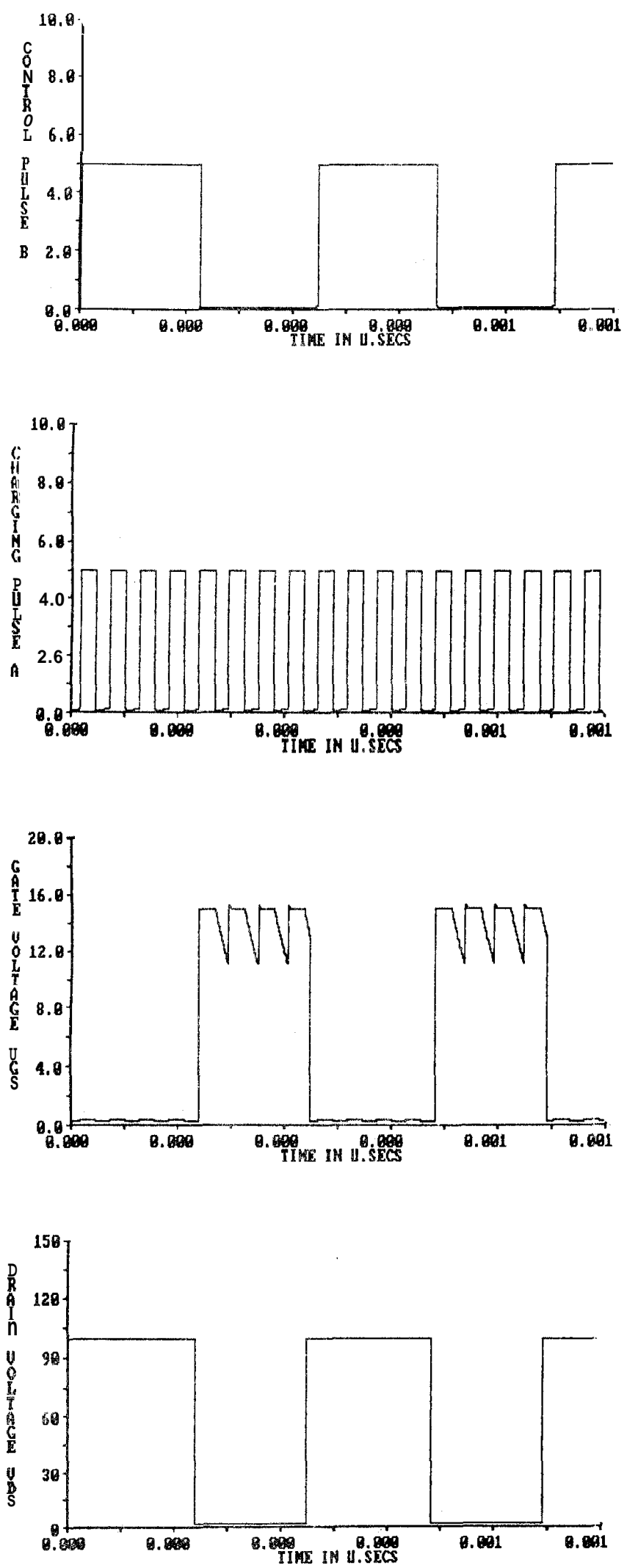

Figure 3: Waveforms from Simulation Results
The gate source capacitance is charged for the duration when $B$ is low and $Q 3$ is in the OFF-state. The charging and discharging of the CGS(eff) with respect to $A$ and $B$ can be seen in Figure 3. At the end of the charging period, when B goes high, Q3 is turned-ON via Q2 and E2 thereby reducing the voltage U belnw the required threshold voltage vo and consequently turning off 04 , It car: be observed that even at the end of the discharging period, when $B$ is low, the voltage at node 10 across the gate source capacitance is above the threshold level and therefore Q4 continues to be in the ONstate with low $R_{D_{S}}(\mathrm{ON})$.

The detailed listing of the PSPICE program is given in the Appendix I which can be run on a personal computer,. The effect of variation of different parameters such as VTO, CGSO, leakage current path resistance R12 and zener clamping voltage represented by VS3, on the switching characteristics of the MOSFET can be studied using PSPICE program before implementing the circuit. The various waveforms of pulse $A_{4}$ B and voltages a node 10 and 11 are shown in Figure 3. The results obtained from simulation have been found to agree well. with the experimental results.

\section{CONCLUSION}

The power MOSFE]. in combination with this new gate drive circuit provides an ideal alternative to the! BJTs and SCRs as a controlled switch at: Jow switching Erequency and mode-rate power level. The problems associated with considerable dedve power requirement of BJTs and/or involved commutation of SCRs have been alleviaced with the technique presented in this paper for the control of power MOSEETs while retaining its excellent switching characteristics and negligible gate power requirements. The PSPICE program listing has been provided to study the salient features of this new gate drive circuit on a personal computer and relevant waveforms of simulation are also presented.

\section{APEENDIX A}

THIS PROGRAM ANALYSIS NEW GATE DRIVE CIRCUIT FOR POWER MOSFETS

VS1 500 DC 15

VS2 120 DC 100

VS3 900 DC 15

R1 $5 \quad 4 \quad 1 \mathrm{~K}$

R2 $43 \quad 8.2 \mathrm{~K}$

R3 $1210 \mathrm{~K}$

R4 $6 \begin{array}{lll}6 & 0 & 8.2 \mathrm{~K}\end{array}$

R5 $6 \begin{array}{llll}6 & 8 & 10\end{array}$

R6 $5 \begin{array}{llll} & 15 & 1 \mathrm{~K}\end{array}$

R7 $1516 \quad 8.2 \mathrm{~K}$

R8 $181710 \mathrm{~K}$

R9 $14 \quad 0 \quad 8.2 \mathrm{~K}$

R10 $14 \quad 13 \quad 1 \mathrm{~K}$

RI1 $12111 \mathrm{~K}$

R $12 \quad 100039 \mathrm{~K}$

D1 $8 \quad 9$ DSWIT

D2 $8 \quad 10$ DSWIT

MODEL DSWIT $\mathrm{D}(\mathrm{RS}=0.1)$

*VINA IS THE CHARGING, PULSE A AT NODE 1

*VINB IS THE CONTROL PULSE B AT NODE 18 


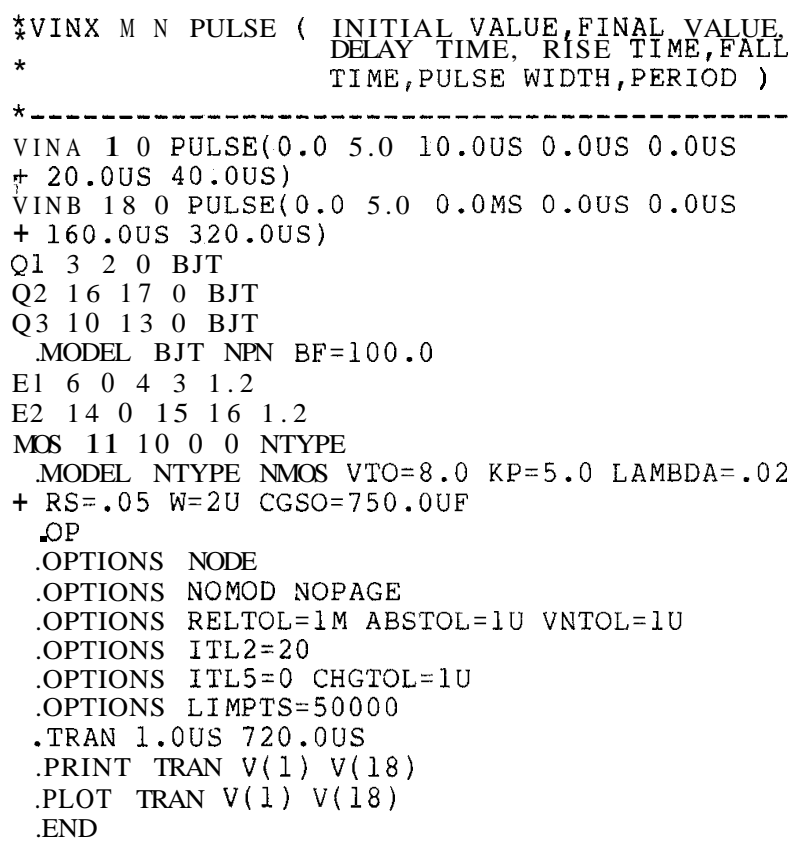

\section{REEEERENCES}

1. Duncan, A. Grant, John Gowar, 'Power MOSFETs - Theory and Applications', John Wiley \& Sons, Inc., (A Wiley Interscience), New York, 1989

2. Betty Prince, Gunrar Due - Gundersen, 'Semiconductor memories', John Wiley \& Sons, Inc., (A Wiley Interscience), New York, 1983 .

3. Walter, A. Tricbel, Alfrea E. Chu, 'Handbook of Semiconductor and Bubble Memories', Prentice-Hall Inc., New Jersey, 1982.

4. Roland Sitting, P. Roggmiller, 'Semiconductor Drives for Power Conditioning', Plenum Press, New York, 1982.

5. H. Nienhans, J. Bowers, 'A high power MOSFET computer model', IEEE Power Electronics Specialists Conf. Record 1980, pp. 97-103.

6. S.R. Narayana Prakash, P.V. Ananda Mohan, B.S.R. Iyengar, Low frequency sinewave inverter using ferrites', Paper identification No. 313, Proc. of the $3 \mathrm{rd}$ European Conf. on Power Electronics and Applications, Aachen, West Germany, Oct. 9$12,1989$.

7. S.R. Narayana Prakash, P.V. Ananda Mohan, B.S.R. Iyengar, 'A new sinewave inverter with high frequency link and synchronous rectification using power MOSFET', IEEE Power Electronics Specialists Conf., San Antonio, Texas, U.S.A., June 11-14, 1990 .

8. S.R. Narayana Prakash, P.V. Ananda Mohan, B.S.R. Iyengar, 'A novel high frequency PMM inverter generates sinusoidal output voltage at $50 \mathrm{~Hz}^{\prime}, \quad$ Presented at the IEEE International Telecommunication Energv Conf., Orlando, Florida, U.S.A., Oct. 2l-2 1990 . 